\title{
SNS CHOICE AND USAGE: A COMPREHENSIVE RESEARCH MODEL
}

\author{
Fang Deng, Hubei University of Education, wpqldf@126.com \\ Zhenyu Huang, Central Michigan University, huang1z@cmich.edu \\ Dalaa Alqahtani, Central Michigan University, alqah1d@cmich.edu \\ James J. Cappel, Central Michigan University, cappe1jj@cmich.edu
}

\begin{abstract}
The use of Social Network Sites has seen tremendous growth in the past years and gained attention and interests from industries. However, with many types of SNS sites available, what factors impact people's choice of SNS and their usage are still not clear. This uncertainty could misguide industries' strategies in SNS and causes wastes and failures in their SNS management. While the extant literature finds factors that influence people's usage of SNS, most of them only focus on one or two aspects. This research however proposes a comprehensive research model that investigates three major categories of factors, namely personal, interpersonal, and cultural that have impacts on both SNS choice and SNS usage systematically. Seven propositions are given to delineate the relationships between these factors and SNS choice and usage. This research sheds lights on research of SNS and makes a good foundation for future empirical research in this regard.
\end{abstract}

Keywords: Social Network Sites, Social Media, Personality, Interpersonal, Cultural, Choice, Usage

\section{INTRODUCTION}

The use of Social Network Sites has been increasingly popular over the years. Social Network Sites (SNS) are websites that allow the user to express themselves, establish their social networks, be able to connect with others, and gain satisfaction [6]. SNS have been a channel for people to connect with others, a means for virtual social interactions and a modern take on forums with various features, such as allowing users to upload diverse content like photos, videos, and personal diaries and network with other SNS users etc. A recent Survey [9] indicated that the percentage of adult internet users who are actively participating in one of the social network web sites has increased from 8 percent in 2005 to 46 percent in 2009 [10].

Popular Social network sites, such as Facebook, has 500 millions of active users, according to the Facebook press (Facebook, 2010). Another popular social network site is Twitter that has a registered number of 175 million users generating traffic of 95 million tweets a day and spending over 110 billion minutes on the SNS [13]. With the rapid increase of SNS usage, and the amount of time that the upcoming generations is spending on SNS, the study on how people use SNS is of great significance. Seeing as the majority of the world's population is interconnected through social network sites, the study of how various personalities, interpersonal interaction styles, and cultures influence the use of SNS. We think the following research questions are of high importance.

RQ1. A holistic research model is needed to investigate the multiple influencing factors together.

RQ2. The significance of these factors on SNS choice and usage vary from being considered alone because of the interaction and influence among these characteristics.

This research will build a theoretical model that incorporates all three factors into consideration when we analyze social media phenomena.

\section{LITERATURE REVIEW}

In order to further look into the user's choice of SNS and how they use it, we propose that cultural, interpersonal, and personal characteristics have effects on a person's choice and use of SNS; and the effects of these factors are not standalone but interact with each other. Students who recently graduated from colleges, for example, would want to expand their networking abilities; they tend to use SNS such as LinkedIn to develop their professional profiles to showcase themselves and appeal to employers. Some people have other kinds of interests in using social network sites. They use SNS such as YouTube, Flicker and Instagram to promote themselves by displaying their different life 
experiences [13]. This being said, different personalities may lead to different means of using SNS. A person who portrays a narcissistic personality traits would want to constantly portray him as a sociable person $[8,16]$. However, the effect of personality on SNS usage is also under the influence of interpersonal factors. A narcissistic person may have to revise or scale down his way of using SNS if he also notices that his SNS connections do not seem to appreciate his social showoff or his SNS postings are not "liked". The effects of personality on SNS use are affected by interpersonal relationships, online or in reality. Another important factor influencing SNS usage is culture. A person with a conservative cultural background may not want to publish their life status online, whereas a person with a more liberal background would like to portray themselves more socially. In order to further look at the various personality traits that a person has when it comes to SNS usage and choice, we divide the characteristics into three categories. The effects of these characteristics have been studied on social media usage more or less. However the extent literature fails to consider these categories of factors together. Due to the interaction described in literature among these, it is critical to consider all factors systematically and holistically when it comes to SNS study.

\section{Personal}

Personal Characteristics of social network users are of abundance. The Big five personality traits, are the major personalities that have been studied and used frequently. The personalities listed are derived from McCrae \& Costa [11], where they first described the Big Five personality characteristics. The Big five consists of Extraversion, Agreeableness, Conscientiousness, Neuroticism, and Openness to Experience. Other personality traits that we also consider are emotional stability or self-esteem, narcissism and sensation seeking $[2,7,8,14,15,16]$.

Various articles investigated how certain personality traits affected the use of SNS such as Facebook, Twitter, Flicker, RenRen and YouTube (e.g. [2,7, 8, 14, 15, 16]). A research conducted toward college students and their usage of SNS shows that students whose personality traits are described as extraversion tend to post things more regularly on their wall, more actively contribute to news feeds, add comments, and like other's posts [8]. Adults with other personalities use Social network sites differently. For example, while extravert adults more likely utilize status updates and comments features, neurotic adults would only use status updates in order to self-express and almost never comment back to other's status postings. SNS users who are of the agreeable personality or high selfesteem would like to comment on other's postings. Those who are of openness and sensation seeking characteristics would use different forms of SNS and like to play online SNS games. Narcissistic users would constantly post images of themselves looking attractive or tend to constantly update their status letting others know about their lives [16]. The following table (Error! Reference source not found.) summarizes personality traits, and their roles in SNS, from extent literature. (X represents characteristics that are significant, $\mathrm{O}$ represents characteristics that are proven not significant).

Table 1. Personal Characteristics and SNS

\begin{tabular}{|c|c|c|c|c|c|c|c|c|}
\hline \multicolumn{9}{|c|}{ Personal Charcteristics } \\
\hline Articles & BF 1 Extraversion & BF 2Agreeableness & BF 3 Conscientiousness & BF 4 Neuroticism & BF 5 Openness to experience & Self esteem & Narcissim & Senstation seeking \\
\hline (Wang, 2012) & $\mathrm{x}$ & $\mathrm{x}$ & $\mathrm{x}$ & $\mathrm{x}$ & $\mathrm{x}$ & $\mathrm{x}$ & $\mathrm{x}$ & $\mathrm{x}$ \\
\hline (Karl, 2010) & & $\mathrm{x}$ & $\mathrm{x}$ & & & $\mathrm{x}$ & & \\
\hline \multicolumn{9}{|l|}{ (Shneor, 2014) } \\
\hline (Wan-Hsiu, 2012) & & & & & & $\mathrm{x}$ & & \\
\hline \multicolumn{9}{|l|}{ (Gong, 2014) } \\
\hline (Lee, 2014) & $\mathrm{x}$ & $\mathrm{x}$ & $\mathrm{x}$ & $\mathrm{x}$ & $\mathrm{x}$ & & $\mathrm{x}$ & \\
\hline
\end{tabular}

\section{Interpersonal}

Social network users possess various interpersonal characteristics. These personal characteristics are sociability, social influence, self-esteem, status, physical dimension, task orientation and perceived enjoyment. Interpersonal characteristics can influence a person's use of social network sites. A person who is sociable is capable of maintaining and developing social relationships online with other people. Also, sociability can influence a SNS user's perceived enjoyment [10]. Status refers to the social status of a person. How a person shares his information on SNS sites would increase their social status in a certain social group. A person with high social status appears more often on SNS sites, or have a more trending SNS social life. Interpersonal characteristics result in on how users use SNS and what choices they decide upon when using them $[1,4,10,12,15)$. The following chart shows the interpersonal characteristics used in the articles. 
Table 2. Interpersonal Characteristics

\begin{tabular}{|l|c|c|c|c|c|}
\hline \multicolumn{4}{|c|}{ Interpersonal Charteristics } \\
\hline Articles & Task Orientation & Social Identity / Scoialability & Physicial Dimension & Status & Social Influence \\
\hline (Bakare, 2012) & $\mathrm{X}$ & $\mathrm{O}$ & $\mathrm{X}$ & $\mathrm{X}$ \\
\hline (Li, 2011) & $\mathrm{X}$ & $\mathrm{X}$ \\
\hline (Pagani, 2011) & $\mathrm{X}$ & $\mathrm{X}$ \\
\hline (Hoffman, 2012) & $\mathrm{X}$ & $\mathrm{X}$ \\
\hline (Wan-Hsiu, 2012) & $\mathrm{X}$ & & \\
\hline (Guo, 2015) & & $\mathrm{X}$ & $\mathrm{X}$ \\
\hline (Lee, 2014) & $\mathrm{X}$ & $\mathrm{X}$ & 0 & \\
\hline
\end{tabular}

\section{Cultural}

A person's cultural background also plays an important role when it comes to a person's usage and choice of SNS. In order to further look at the cultural influences, we are using Hofstede's cultural characteristics- individualism, uncertainty avoidance, masculinity, power distance and long-term orientation. Other cultural characteristics that we investigate include intellectual anatomy, mastery and egalitarianism.

A person whose cultural values promote them to be more individualistic and self-dependent uses SNS differently than those where cultures are male dominant. A culture that is more intellectually anatomic and promotes individuals to follow their own ideas, may reuse SNS differently. According to Gong, masculinity can be negatively associated with the use of SNS. Whereas Wan-Hsiu finds individualistic societies have a high independence value and therefore behave differently than with a more collective society $[2,7,8,14,15,16]$. The following table identifies the various cultural characteristics studied by SNS research articles reviewed.

Table 3. Cultural Influences

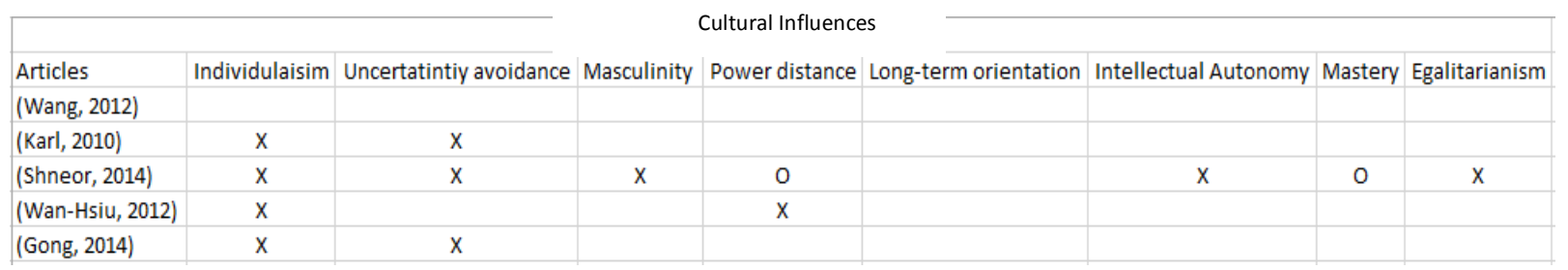

\section{Summary}

The choice of SNS is impacted by the personality traits. The Choice of SNS refers to that a network site is adopted by a user for professional or personal usage and opens an account on this SNS. The usage of SNS is defined by the time a user spends on SNS, how actively that person maintains his SNS status and how frequently he interacts with others via the SNS.

The use of SNS can be influenced by many characteristics as defined, which play an important role in users' decision on SNS selection and the way they use SNS. A user who is an Extrovert (Personal Characteristic), high social identity (Interpersonal Characteristic) and Individualistic (cultural Characteristic) would use social network sites more actively than an individual who is introverted, less social, and conservative. A SNS user will choose a SNS that is more compatible with their personality and would interact via that SNS more actively than on the SNS adopted because of peer pressure. He would also be more active on SNS than a person who is task oriented (Interpersonal characteristic), Conscientious (Personal characteristics), and mastery (cultural Characteristic). Most importantly, we believe these three categories of factors do not act independently on the SNS choice and usage. They interact with each other. The personality can be enhanced or inhibited by a SNS user's interpersonal relationship online and his cultural background. When we consider one aspect of factors alone like most SNS research has done we may miss the big picture. A person's cultural relation and perception can be changed by the 
exposure to the enormous virtual SNS world. His personality and interpersonally interaction is under impact and subject to changes. These changes in in turn influence his SNS choice and usage. The following comprehensive research model (Figure 1) is constructed to demonstrate the relationship between three important categories of factors and SNS choice and usage.

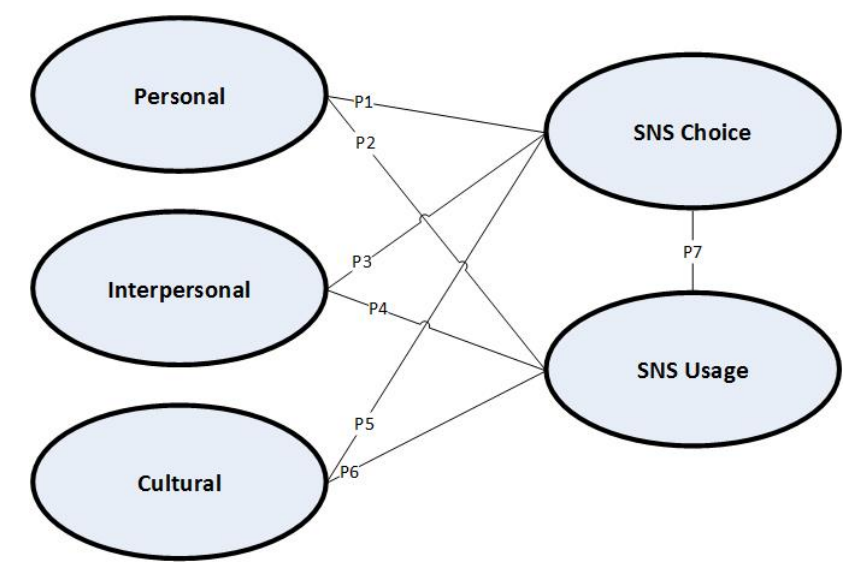

Figure 1. Comprehensive Research model on SNS Choice and Usage

\section{Theoretical Model and Propositions}

Based on the comprehensive research model, we propose following seven propositions to explain the relationship between the three categories of factors and SNS choice and SNS usage. These propositions are based on analysis of extent literature and theoretical speculation. They make a good foundation for future empirical studies that will develop hypotheses from these propositions and collect data to prove those hypotheses.

\section{Proposition 1 Personal Characteristics have significant effects on SNS Choice}

The choice of SNS is determined by a user's character. A user can easily find out that if the design of a SNS is compatible with his personality or not. The features supported by a SNS may or may not be liked by a person. A person who is more outgoing may choose a SNS that is more socially interactive, allows him to outreach more people he might not interact in real life, allows him to post things to a greater audience.

\section{Proposition 2 Personal Characteristics have significant effects on SNS Usage}

People's personal characteristics determine the use of SNS sites, and their way of using them based upon how open they are to experiences. If they are conscientious they would be more aware of what they post on SNS, and who they follow. A user may adopt SNS for many reasons. Self-interesting, peer pressure, current trend, news reporting etc. all can drive a person to adopt a 'popular' SNS. However, the usage is closely related to his personality. An opened account on a SNS does not necessarily mean that the user would use the SNS.

\section{Proposition 3 Interpersonal Characteristics have significant effects on SNS Choice}

The relationship between people and the traits of interpersonal relationship have impact on people's choice of SNS. Some SNS can facilitate interpersonal communication, some are not perceived so. Therefore considering the Interpersonal Characteristics will help determine a user's choice of SNS. One ambitious person may favor a SNS for professional development, whereas others with a low self-esteem may choose a social network that makes them feel relaxed with the least amount of social interaction. 


\section{Proposition 4 Interpersonal Characteristics have significant effects on SNS Usage}

This proposition depicts, how people interact with other people, how close they are to each other, and how often they interact online have impacts on their use of social network. The Interpersonal Characteristics in this proposition help elaborate why SNS users who value ethics and morality tend to use social network sites in a more cautious way, whereas those who are task oriented tend to focus on a more professional social network site that has a specific and dedicated purpose.

\section{Proposition 5 Cultural Characteristics have significant effects on SNS Choice}

SNS choice also is influenced by cultural values. A SNS is an extension of a society where culture normally plays key roles. However, the virtual nature makes a SNS sometimes free from the control of a culture. Users who have a more individualistic culture, may choose a SNS site that is more open to choice and experience, whereas someone who comes from a masculine culture, may want to choose to participate in a social network that is to their cultural values. A conservative culture may make a user choose more text-based SNS than picture or video oriented ones.

\section{Proposition 6 Cultural Characteristics have effects on SNS Usage}

As people come from different cultural backgrounds socialize on a SNS, cultures may cause conflicts and unease and thus play important roles in extent of SNS usage. Social network users who come from a more conservative background may tend to use social networks less than those who come from a more liberal background. Also, we can see that a society that emphasizes masculinity and is more male dominant, may not allow the women to use SNS or even to express themselves freely on SNS.

\section{Proposition 7 SNS Choice have a significant influence on SNS Usage}

The choice of SNS determines the way a user uses the SNS. A person's choice is significant to the SNS usage, since not only do the characteristics determine the usage of these sites, but why they choose a SNS determines the use. A freely chosen SNS would support frequent usage than those adopted due to peer pressure.

To investigate the effects of personal, interpersonal, and cultural factors on SNS choice and usage further, we select the following traits in each category and discuss their values in depth. According to our literature analysis, big five factors plus self-esteem and sensation seeking are important personality traits that have significant impacts on people's choice of SNS and their usage. The literature review conducted also allowed us to determine the interpersonal characteristics that impact SNS usage and SNS choice. The interpersonal characteristics that are of significance are Task Orientation, Sociability, Physical Dimension, Status, Self- esteem, Social Influence and Perceived Enjoyment. Furthermore according to our literature review Cultural Characteristics such as Hofstede's cultural dimension and Intellectual Anatomy, Egalitarianism and Mastery are used to determine the use of SNS, and the choice of SNS. Therefore our model illustrates the various characteristics in each category, and how they are applied in the theory.

\section{CONCLUSIONS}

The presented research identifies how the significance of a person's characteristics affects their usage of SNS and the choice related to it. We believe that the choice of Social network sites is of significance in the research because a person's characteristics signify the choice of SNS, which in turn also impacts SNS usage. Proposition 7 is addresses this relationship when considering the freedom of SNS choice as a motivator and incentive for the use of that SNS. Figure 2 extends the theoretical model delineated in figure 1, by listing out the key factors from each category. This provides for a better guidance to future research that will investigate SNS choice and usage empirically.

Additionally, future studies can allow businesses to use the characteristics specified in order to target the audience they want to sell or promote their products. Social network sites are a new way for businesses to find their potential customers and if they can interact with the customers properly, they are able to reach out to a wider audience. Industries need to consider a user's personal, interpersonal, and cultural factors to develop a suitable SNS strategy. 


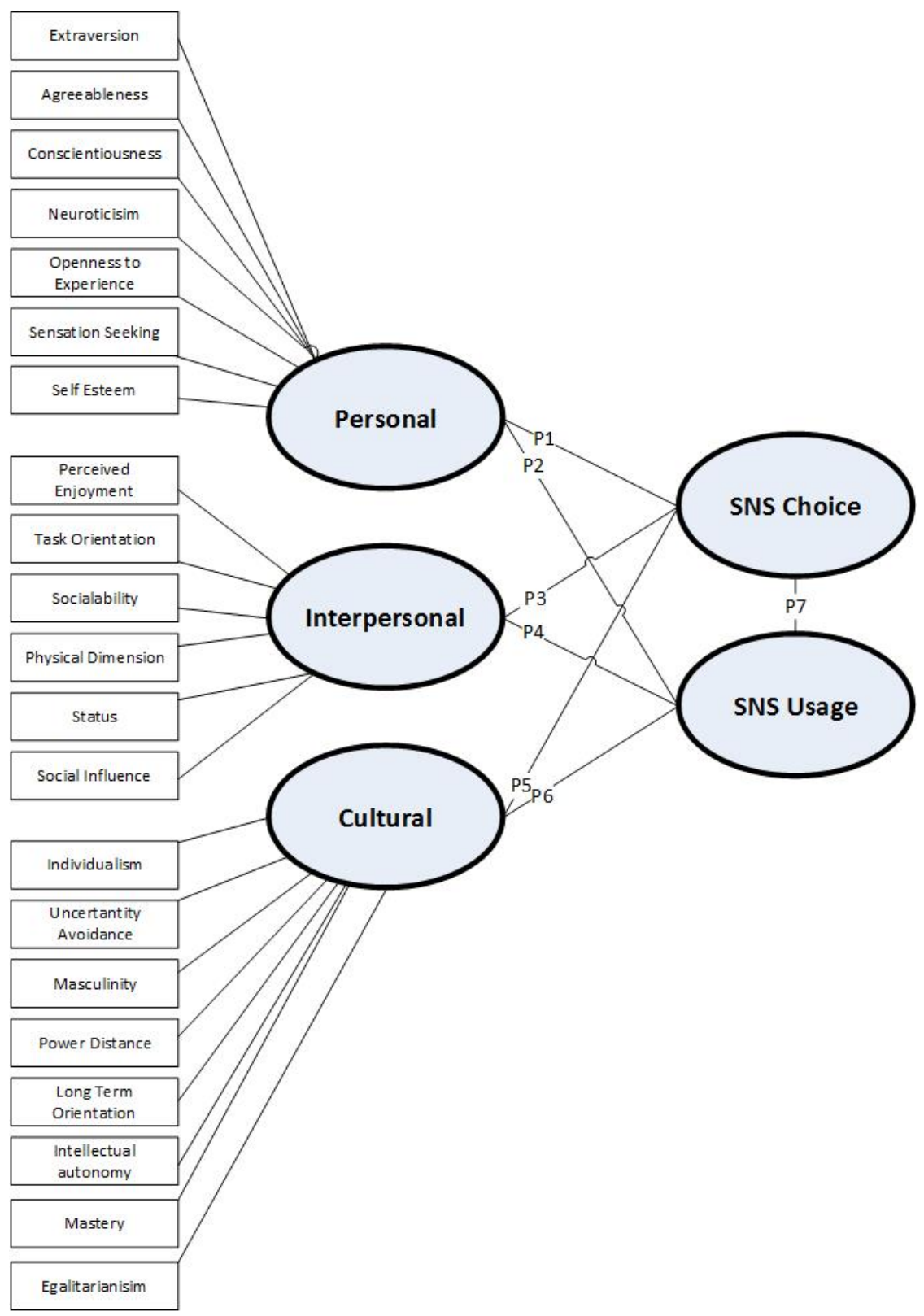

Figure 2. Research Model Expanded

\section{REFERENCES}

1. Bakare, K. K., Zamzami, I. F., \& Olowolayemo, A. (2012). Students' orientation towards interpersonal communication in online social networking sites. Multicultural Education \& Technology Journal, 6(1), 3644.

2. Gong, W., Stump, R. L., \& Li, Z. G. (2014). Global use and access of social networking web sites: A national culture perspective.Journal of Research in Interactive Marketing, 8(1), 37-55.

3. Guo, H., Pathak, P., \& Cheng, H. K. (2015). Estimating social influences from social networking sites articulated friendships versus communication interactions. Decision Sciences, 46(1), 135. 
4. Hoffman, D. L., \& Novak, T. P. (2012). Why do people use social media? empirical findings and a new theoretical framework for social media goal pursuit Rochester: Social Science Research Network.

5. Ji, Y., Wang, G. J., Zhang, Q., \& Zhu, Z. H. (2014). Online social networking behaviors among Chinese younger and older adolescent: The influences of age, gender, personality, and attachment styles. Computers in Human Behavior, 41, 393-402.

6. Joseph, B., Walther, B., Van Der, H., Sang-Yeon, K., David, W. and Stephanie, T.T. (2008), The role of friends' appearance and behavior on evaluations of individuals on Facebook: are we known by the company we keep?, Human Communication Research, Vol. 34, pp. 28-49.

7. Karl, K., Peluchette, J., \& Schlaegel, C. (2010). Who's posting facebook faux pas? A cross-cultural examination of personality differences. International Journal of Selection and Assessment, 18(2), 174-186.

8. Lee, E., Ahn, J., \& Kim, Y. J. (2014). Personality traits and self-presentation at Facebook. Personality and Individual Differences, 69, 162-167.

9. Lenhart, A. (2007). Cyberbullying. Pew Internet \& American Life Project. $<$ http://www.pewinternet.org/Reports/2007/Cyberbullying.aspx $>$.

10. Li, D. C. (2011). Online social network acceptance: A social perspective. Internet Research, 21(5), 562580.

11. McCrae, R. R., \& Costa,Paul T.,„Jr. (1987). Validation of the five-factor model of personality across instruments and observers.Journal of Personality and Social Psychology, 52(1), 81.

12. Pagani, M., Hofacker, C. F., \& Goldsmith, R. E. (2011). The influence of personality on active and passive use of social networking sites. Psychology \& Marketing, 28(5), 441.

13. Pérez, M., \& Gómez, J.,M. (2011). Why do people use social networks? Communications of the IIMA, 11(2), 41-49.

14. Shneor, R., \& Efrat, K. (2014). Analyzing the impact of culture on average time spent on social networking sites. Journal of Promotion Management, 20(4), 413.

15. Wan-Hsiu (Sunny) Tsai, \& Linjuan, R. M. (2012). Cultural values reflected in corporate pages on popular social network sites in china and the united states. Journal of Research in Interactive Marketing, 6(1), 4258.

16. Wang, J. L., Jackson, L. A., Zhang, D. J., \& Su, Z. Q. (2012). The relationships among the Big Five Personality factors, self-esteem, narcissism, and sensation-seeking to Chinese University students' uses of social networking sites (SNSs). Computers in Human Behavior, 28(6), 2313-2319. 